\title{
New Approach to Approximate Circular Arc by Quartic Bezier Curve
}

\author{
Azhar Ahmad ${ }^{1}$, Rohaidah Masri, \\ Nor'ashiqin Mohd Idrus \\ Department of Mathematics \\ Universiti Pendidikan Sultan Idris \\ Tanjung Malim, Perak, Malaysia \\ 1'azhar.ahmad@fsmt.upsi.edu.my
}

\author{
Jamaluddin M. Ali \\ School of Mathematical Science \\ Universiti Sains Malaysia \\ Penang, Malaysia
}

\begin{abstract}
This paper presents a result of approximation an arc circles by using a quartic Bezier curve. Based on the barycentric coordinates of two and three combination of control points, the interior control points are determined by forcing the curvature at median point as similar as the given curvature at end points. Hausdorff distance is used to investigate the order of accuracy compare to the actual arc circles through central angle of $0 \leq \theta<\pi$. We found that the optimal approximation order is eight which is somewhat similar to preceding methods in the literatures.
\end{abstract}

Keywords- arc circle, barycentric coordinate, Hausdorff distance, quartic Bezier

\section{Introduction}

Parametric representations of curves and surfaces are essential to the field of Computer Aided Geometric Design (CAGD). Representing of an arc circles using parametric curves is one of an important study in related to design conic sections in geometric modeling. The important of arc circles in geometric modeling are undeniable, along with the used of the others primitive elements, such as a straight line, conic curves and spiral. Arc circles are widely used in CAD/CAM and can be found in industrial as well as in product manufacture; the aircraft and the automobile industries where surfaces are frequently constructed from curves involving circular arcs using lofting or skinning technique [4]. Furthermore it is used in solving communication problem between $\mathrm{CAD} / \mathrm{CAM}$ systems when working with curve representations in different data formats [8]. Generally, these significant uses of parametric polynomial curve are concentrated on the aesthetical and functional purposes. Since NURBS form is the standard form in most of geometric software systems so the used of the curve that based on parametric polynomial representation became more important.

A considerable amount of work has been done on approximating circular arc by polynomial curves. We consider that most of the previous work related to the used of the low degrees of Bezier curve modeling. Although a circle arc can be exactly represented by rational parametric curve of low degree, such the rational quadratic Bezier, some CAD/CAM systems require a polynomial representation of circular segments. Also, some important algorithms, such as lofting and blending cannot be directly applied to rational curves [10]. Previous work showed that we can only representing an arc circle by polynomials curve with a small acceptable tolerance. The cubic approximation was proposed by de Boor et al. [2] and Dokken et al. [3]. Goldapp [5] presented the best $G^{k}$ cubic approximations for $k=0,1,2$, whose approximation orders are six. Ahn and Kim [1] gave the quartic $G^{3}$ approximation to arc of angle $0 \leq \theta<\pi$. Their approach has the optimal approximation of order eight. And they also find the quintic $G^{4}$ approximation with order ten. Kim and Ahn [7] presented the quartic Bezier approximations of circular arcs with approximation of order eight by using subdivision scheme with equi-distance of the circular arc. The approximation proposed by them has a smaller error than other previous results. By using quintic, Fang [4] presented a scheme with different boundary conditions that yields approximation curves with $G^{k}, k=2,3,4$ continuities at the circular arc's ends.

In our method, we consider the barycentric coordinate with positive ratio to locate the interior control points of the quartic form and suggest the symmetrical position of first two points against the last two points respect to the middle point. From this assumption, we can automatically avoid the existence of cusp, loop and the inflexion point. We suggest a simple and direct method to obtain those approximations which facilitate to designers. In practice, designers or users usually do not concern about the underlying mathematics and related equations. This paper only discusses the curves with second order of geometric continuity and not extended on higher order since it is adequate for our objective. Since it involved the manipulation of quartic form, this will involve a long and difficult mathematical computation, so the use of CAS manipulator such as MATEMATICA will make a great help.

The remaining part of this paper is organized as follows. We start with some preliminary background and notation in Section II. We prescribe the method that used to construct an arc circles in the given range of the central angle $\theta \in[0, \pi)$ in Section III. In Section IV, we discuss the radial approximation error and approximation order which based on Hausdorff distance. The 
result is compared to the others best known methods. We present the result of a numerical example in Section V. Finally, we conclude our results in the final section.

\section{Some Preliminary}

The following notations and conventions are used. We write the dot product of two vectors, $A$ and $B$ as $A \bullet B$. The notation of $A \wedge B$ represents the outer product of two plane vectors $A$ and $B$. Note that the dot and outer products are scalar which are written as $A \bullet B=\|A\|\|B\| \cos \theta$ and $A \wedge B=\|A\|\|B\| \sin \theta$, respectively, where $\theta$ is the turning angle from $A$ to $B$. Positive angle is measured anticlockwise. The Euclidean norm or length of vector $A$ is $\|A\|$.

Planar quartic Bezier curve $Z:[0,1] \rightarrow \mathbb{R}^{2}$ is represented as

$$
Z(t)=\sum_{i=0}^{4} P_{i}\left(\begin{array}{l}
4 \\
\mathrm{i}
\end{array}\right)(1-t)^{4-i} t^{i}, \quad 0 \leq t \leq 1
$$

where $P_{i}, i=0,1,2,3,4$ are the control points, $\left(\begin{array}{l}4 \\ \mathrm{i}\end{array}\right)(1-t)^{4-i} t^{i}$ is the Bernstein polynomial and $t$ is global parameter. We denote the end points as $P_{0}$ and $P_{4}$, also $P_{j}$, $j=1,2,3$ as the interior points. As a planar parametric curve, the signed curvature of $Z(t)$ is defined as in [9]:

$$
\kappa(t)=\frac{Z^{\prime}(t) \wedge Z^{\prime \prime}(t)}{\left\|Z^{\prime}(t)\right\|^{3}}
$$

The signed radius is the reciprocal of (2). It is known that $\kappa(t)$ is positive sign when the curve segment bends to the left and it is negative sign if it bends to the right at $t . Z^{\prime}(t)$ and $Z$ " $(t)$ are first and second derivatives of (1).

\section{Prescribing Methods}

In this section, we describe an approach for approximating the given data. The problem that we considered can be expressed in following problem statement;

Let $P_{0}$ and $P_{4}$ be the two points on the circumference of circle with radius $r$, and $T_{0}$ and $T_{1}$ be the unit tangent vectors, respectively. The central angle of this two point is in the interval of $0 \leq \theta<\pi$. Find an approximation of a circular arc that satisfies this given data by using a single Bezier quartic curve.
Firstly, we denote $P_{s}$ as the intersection point between two tangent lines parallel to unit vector $T_{i}, i=0,1$, that pass through endpoints $P_{0}$ and $P_{4}$, respectively. Here, we only consider the construction of curve segment that is negative curvature, whereby for approximation of arc circle with positive curvature is in the similar manner. According to Grandine and Hogan [6], $P_{S}$ can be achieved by

$$
P_{S}=\frac{\left(P_{4} \wedge T_{1}\right) T_{0}+\left(T_{0} \wedge P_{0}\right) T_{1}}{T_{0} \wedge T_{1}}
$$

If we denoted $B_{0}$ as the centre of the circle, so the circular arc is divided symmetrically by line $P_{S} B_{0}$. By using the barycentric coordinate, the interior points $P_{1}, P_{2}, P_{3}$ may now be written in the terms of $P_{0}, P_{4}, P_{5}$ as

$$
\begin{gathered}
P_{1}=(1-\rho) P_{0}+\rho P_{S}, \\
P_{3}=(1-\rho) P_{4}+\rho P_{S}, \\
P_{2}=\alpha P_{1}+\alpha P_{3}+(1-2 \alpha) P_{S} .
\end{gathered}
$$

Point $P_{1}$ and $P_{3}$ are in barycentric form of 2 points, where their barycentric coordinates were $(\rho, 1-\rho)$ and $(1-\rho, \rho)$, respectively. While $P_{2}$ is a barycentric combination of 3 points with coordinate $(\alpha, \alpha, 1-2 \alpha)$. All of those coefficients satisfy $0<\alpha, \rho<1$. Without any loss of generality in terms of translation, rotation, reflection and uniform scaling, we can allocate $P_{0}, P_{4}$ on same ordinate, while $P_{S}$ on $y$-axis and the center of the circle $B_{0}$ on the origin. Fig. 1, shows the position of these control points and their interior points $P_{1}, P_{2}, P_{3}$ with respect to the canonical positions.

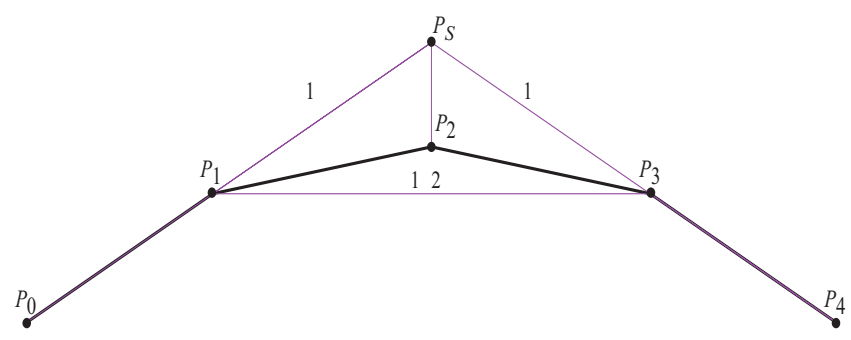

Fig. 1 Position of control points and their barycentric coordinates

Secondly, since our objective is to obtain the sufficient condition of a single segment of Bezier quartic to approximate arc circle with $G^{2}$ continuity condition, hence we fix $\kappa(0)=\kappa(1)=-1 / r$ and forced the curvature at median point as $\kappa(0.5)=-1 / r$. Furthermore, we apply $B_{0}=(0,0)$ and 
$r=1$ since this do not give any effect on $\rho$ and $\alpha$ in general on curvature analysis. The following procedure shows in detail how the curvature is obtained. It starts by letting

$$
\begin{aligned}
& P_{S}=B_{0}+v\left(T_{0}-T_{1}\right), \\
& P_{0}=B_{0}-r N_{0}, \\
& P_{4}=B_{0}-r N_{1},
\end{aligned}
$$

where $v$ is a constant. $N_{0}$ and $N_{1}$ are unit normal vectors of $T_{0}$ and $T_{1}$ at $P_{0}$ and $P_{4}$, respectively. Since we considering the arc circular of negative curvature then $T_{0} \wedge N_{0}<0$ and $T_{1} \wedge N_{1}<0$. Substituting (4), (5) into (1) give $Z(t)$ in the terms of unit vectors as

$$
Z(t)=a_{1} N_{0}+a_{2} N_{1}+a_{3} T_{0}+a_{4} T_{1}
$$

where

$$
\begin{aligned}
& a_{1}=r(-1+t)^{2} . \\
& \left(-1+t^{2}(3+6 \alpha(-1+\rho)-4 \rho)+t(-2+4 \rho)\right) \\
& a_{2}=r t^{2}\left(\begin{array}{r}
t^{2}(3+6 \alpha(-1+\rho)-4 \rho) \\
+6 \alpha(-1+\rho)-4 t(-1+3 \alpha)(-1+\rho)
\end{array}\right), \\
& a_{3}=2(-1+t) t v\left(\begin{array}{r}
t^{2}(3+6 \alpha(-1+\rho)-4 \rho)-2 \rho \\
+t(-3-6 \alpha(-1+\rho)+4 \rho)
\end{array}\right), \\
& a_{4}=-a_{3} .
\end{aligned}
$$

The first derivative of $Z(t)$ is

$$
Z^{\prime}(t)=a_{1}{ }^{\prime} N_{0}+a_{2}{ }^{\prime} N_{1}+a_{3}{ }^{\prime} T_{0}+a_{4}{ }^{\prime} T_{1} .
$$

And the second derivative of $Z(t)$ is

$$
Z^{\prime \prime}(t)=a_{1} " N_{0}+a_{2} " N_{1}+a_{3} " T_{0}+a_{4} " T_{1} \text {. }
$$

Here, $a_{j}{ }^{\prime}$ and $a_{j}{ }^{\prime \prime}$ are first and second derivatives of $a_{j}$, $j=1,2,3,4$, respectively. By using the prior conventions, several product vectors and the related coefficient $v$ is obtained as follow

$$
\begin{aligned}
& \begin{aligned}
N_{0} \bullet N_{0}=T_{0} \cdot T_{0}=N_{1} \bullet N_{1} \\
\quad=T_{1} \cdot T_{1}=N_{0} \wedge T_{0}=N_{1} \wedge T_{1}=1
\end{aligned} \\
& \begin{aligned}
N_{0} \cdot T_{0}= & N_{1} \cdot T_{1}=N_{0} \wedge N_{0} \\
& =T_{0} \wedge T_{0}=N_{1} \wedge N_{1}=T_{1} \wedge T_{1}=0
\end{aligned} \\
& N_{0} \cdot T_{0}=-N_{1} \cdot T_{0}=-\left(T_{0} \wedge T_{1}\right)=-\left(N_{0} \wedge N_{1}\right)=\sin \theta, \\
& T_{0} \cdot T_{1}=N_{0} \bullet N_{0}=N_{0} \wedge T_{1}=N_{1} \wedge T_{0}=\cos \theta, \\
& v=\frac{P_{4} \wedge T_{1}}{T_{0} \wedge T_{1}}=\frac{-r N_{1} \wedge T_{1}}{T_{0} \wedge T_{1}}=\frac{r}{\sin \theta}
\end{aligned}
$$

By applying (8), (9) and (10) onto (2), the numerator and denominator of curvature, $\kappa$ can be write as

$$
\begin{aligned}
& Z^{\prime} \wedge Z^{\prime \prime}=a_{1}{ }^{\prime} a_{3}{ }^{\prime \prime}+a_{2}{ }^{\prime} a_{4} "-a_{4}{ }^{\prime} a_{2}{ }^{\prime \prime}-a_{3}{ }^{\prime} a_{1} " \\
& +\left(a_{2}{ }^{\prime} a_{1}{ }^{\prime \prime}-a_{1}{ }^{\prime} a_{2}{ }^{\prime \prime}\right) \sin \theta+\left(a_{2}{ }^{\prime} a_{3}{ }^{\prime \prime}-a_{3}{ }^{\prime} a_{2}{ }^{\prime \prime}\right) \cos \theta \\
& +\left(a_{4}{ }^{\prime} a_{3}{ }^{\prime \prime}-a_{3}{ }^{\prime} a_{4}{ }^{\prime \prime}\right) \sin \theta+\left(a_{1}{ }^{\prime} a_{4}{ }^{\prime \prime}-a_{4}{ }^{\prime} a_{1}{ }^{\prime \prime}\right) \cos \theta
\end{aligned}
$$

$$
\begin{aligned}
& Z^{\prime} \cdot Z^{\prime}=\left(a_{1}^{\prime}\right)^{2}+\left(a_{2}^{\prime}\right)^{2}+\left(a_{3}\right)^{2}+\left(a_{4}{ }^{\prime}\right)^{2} \\
& +2\left(a_{1}{ }^{\prime} a_{4}{ }^{\prime}-a_{2}{ }^{\prime} a_{3}{ }^{\prime}\right) \sin \theta+2\left(a_{1}{ }^{\prime} a_{2}{ }^{\prime}-a_{3}{ }^{\prime} a_{4}{ }^{\prime}\right) \cos \theta
\end{aligned}
$$

Since as fixed earlier that $\kappa(0)=\kappa(1)=-1$, we achieve

$$
\frac{3 \alpha(-1+\rho) \cos ^{3} \frac{\theta}{2} \sin \frac{\theta}{2} \csc \theta}{\rho^{2}}=-1 .
$$

And as $t=1 / 2$, so for $\kappa(1 / 2)=-1$, simplification yield to

$$
\frac{12(1+2 \alpha(-1+\rho))(-3+2 \rho) \sin \frac{\theta}{2} \csc \theta}{(3-2 \rho)^{3}}=-1 \text {. }
$$

From (13), $\alpha$ can be write in the terms of $\rho$ and $\Phi=\theta / 2$ as

$$
\alpha=-\frac{2 \rho^{2} \sec ^{2} \Phi}{3(-1+\rho)} \text {. }
$$

Substituting (15) into (14), we get the quadratic equation in the terms of $\rho$ as follows

$$
\rho^{2}\left(-4-8 \sec ^{3} \Phi\right)+12 \rho-(9-6 \sec \Phi)=0
$$

It is clear from (16) that if $\cos \Phi \geq \frac{2}{3}$ then we get two positive values of $\rho$. The result is obtained from $-(9-6 \sec \Phi) \leq 0$ which come directly from Descartes's Rule of Signs. And from the discriminate of (16) we prove that there exist two real values as shown below

$$
96(-1+\sec \Phi)^{2}(1+2 \sec \Phi) \sec \Phi \geq 0 .
$$


If we name $\rho_{1}, \rho_{2}$ as the solutions of (16), we obtain

$$
\begin{aligned}
& \rho_{1}=\frac{3-\sqrt{6} \sqrt{(-1+\sec \Phi)^{2}(1+2 \sec \Phi) \sec \Phi}}{2+4 \sec ^{3} \Phi}, \\
& \rho_{2}=\frac{3+\sqrt{6} \sqrt{(-1+\sec \Phi)^{2}(1+2 \sec \Phi) \sec \Phi}}{2+4 \sec ^{3} \Phi} .
\end{aligned}
$$

Finally, by substituting each of these solutions into (15), we obtain the values of $\alpha$ that satisfies (13) and (14). As the result, $\left(\rho_{1}, \alpha_{1}\right)$ and $\left(\rho_{2}, \alpha_{2}\right)$ are two possible values that give us the necessary condition to approximate the given problem. Fig. 2 shows the function of $\rho_{1}$ and $\rho_{2}$ given in plain and dashed curves, respectively, which referred to $0 \leq \Phi<\pi / 2$. Positive value of $\rho_{1}$ is $[0,0.5]$ since interval of $\Phi$ is $\left[0, \cos ^{-1}(2 / 3)\right]$.

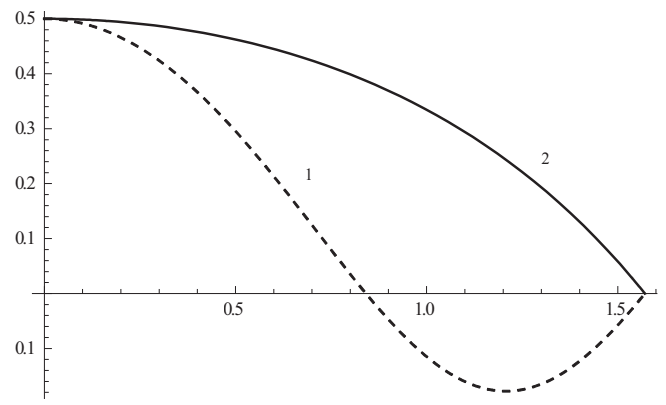

Fig. 2 Value of $\rho_{1}$ and $\rho_{2}$ for $0 \leq \Phi<\pi / 2$

Since $\rho_{2}$ has bigger range of $\Phi$ compare to his counterpart $\rho_{1}$, so for further discussion we only consider $\rho_{2}$ where we obtain an approximate curve which satisfies the given problem in high order of accuracy. The explicit approximate quartic form which is easy to use can been stated as follows.

An approximation quartic Bezier of circular arc can be found by using (1), with control points as (4) and (3), where $\rho$ as (19), $\alpha$ from (15) and the central angle is

$$
\theta=\left\{\begin{array}{ccc}
\left|\sin ^{-1}\left(T_{0} \wedge T_{1}\right)\right| & \text { if } & T_{0} \cdot T \geq 0 \\
\pi-\left|\sin ^{-1}\left(T_{0} \wedge T_{1}\right)\right| & \text { if } & T_{0} \cdot T<0
\end{array}\right.
$$

It is clear that the parameters $\alpha$ and $\rho$ are depend on the central angle that has been created by the end points. Here, $\theta$ as specified in (20) satisfy the acute and obtuse angle.

\section{Approximation Accuracy Analysis}

In this section we discuss the accuracy of the approximation that make by the quartic Bezier form which introduced in the previous section. The approximation accuracy is obviously considered as a distance between set of points on given curves, i.e., a circular arc and a quartic curve in this case. Here we will consider Hausdroff distance as proposed by Ahn and Kim [1], where it is most reliable scheme to measures how far two subsets of a Euclidean space are from each other. The Hausdorff distance can be described as the longest distance between two ste of points. First, let us consider $b$ as the arc circle, so the Hausdroff distance between the arc circle and the approximation arc can be derived as follows

$$
d_{H}(b, Z)=\max _{t \in[0,1]}|\varepsilon(t)|
$$

where

$$
\varepsilon(t)=\sqrt{x^{2}(t)+y^{2}(t)}-1
$$

The function in (22) is known as the radical error, and another alternative error function that has been used for the analysis is

$$
\psi(t)=x^{2}(t)+y^{2}(t)-1 .
$$

Function (22) and (23) have their zero sets and extreme values at the same locations, however using (23) is preferable since there is no square root term involved. It can be shown that when $\psi(t)$ is small, then $\psi(t) \approx 2 \varepsilon(t)$ as in [4]. We use those two error functions in order to determine the approximate errors and for comparisons manner.

We locate the end points of arc circle at $P_{1}=(1,0)$, $P_{4}=(\cos \Phi, \sin \Phi)$ with center of the unit circle is $B_{0}=(0,0)$. Since we consider the minor arc circle with positive curvature therefore the unit tangent vectors at $P_{0}, P_{4}$ are $T_{0}=(0,1)$ and $T_{1}=(-\sin \Phi, \cos \Phi)$, respectively. As a result of substituting $P_{S}=r\left(T_{0}-T_{1}\right) / \sin 2 \Phi$ and (4) into (1), the components of quartic curve $Z(t)$ is simplified as

$$
\begin{aligned}
x(t)= & (-1+t)^{4}-4(-1+t)^{3} t+t^{4} \cos 2 \Phi \\
- & 6(-1+t)^{2} t^{2}(-1+\alpha-\alpha \rho+\alpha(-1+\rho) \cos 2 \Phi) \\
& +4(1-t) t^{3}(\rho+\cos 2 \Phi-\rho \cos 2 \Phi),
\end{aligned}
$$

and

$$
\begin{aligned}
& y(t)= \\
& t\left(\begin{array}{l}
t^{3} \sin 2 \Phi-4(-1+t)^{3} \rho \tan \Phi \\
-6(-1+t)^{2} t(-1+\alpha-\alpha \rho+\alpha(-1+\rho) \cos 2 \Phi) \tan \Phi \\
-4(-1+t) t^{2}(\sin 2 \Phi-\rho \cos 2 \Phi \tan \Phi)
\end{array}\right) .
\end{aligned}
$$

So $\psi(t)$ and it's derivative $\psi^{\prime}(t)$, respectively are

$$
x^{2}+y^{2}-1=B(-1+t)^{3} t^{3}\left(\left(t-\frac{1}{2}\right)^{2}-k\right),
$$


and

$$
\left(x^{2}+y^{2}-1\right)^{\prime}=B(-1+t)^{2} t^{2}(-1+2 t)\left(\left(t-\frac{1}{2}\right)^{2}-h\right)
$$

where

$$
\begin{gathered}
B=\tau^{2} \sec ^{2} \Phi \tan ^{4} \Phi, \\
k=\frac{\tau^{2}+8 \omega \cos ^{2} \Phi}{4 \tau^{2}}, \\
h=\frac{\tau^{2}+6 \omega \cos ^{2} \Phi}{4 \tau^{2}}, \\
\tau=-3+4 \rho+8 \rho^{2}+(-3+4 \rho) \cos 2 \Phi, \\
\omega=\left(-3+15 \rho-16 \rho^{2}\right. \\
-16 \rho^{3}+4\left(-1+4 \rho-4 \rho^{2}+4 \rho^{3}\right) \cos 2 \Phi \\
+(-1+\rho) \cos 4 \Phi .
\end{gathered}
$$

As $\psi(t)=0$, we have zeros with multiplicity 3 at $t=0$, $t=1$, and each at $t=1 / 2 \pm \sqrt{k}$ with total zeros are 8 . While as $\psi^{\prime}(t)=0$, the zeros is obtained at $t=0$ and $t=1$ with multiplicity $2, t=1 / 2$ and $t=1 / 2 \pm \sqrt{h}$ with total zeros are 7 . Therefore, the extremum value of $\psi(t)$ happen at $t=1 / 2$, since both $t=1 / 2 \pm \sqrt{h}$ and $t=1 / 2 \pm \sqrt{k}$ are complex roots due to negative values of $h$ and $k$. This conjecture can be show from the limits analysis as below.

$$
\lim _{\Phi \rightarrow 0} \rho=\frac{1}{2}, \lim _{\Phi \rightarrow 0} h=-\infty \text {, and } \lim _{\Phi \rightarrow \pi} \rho=0, \lim _{\Phi \rightarrow 0} h=-\infty .
$$

In the similar manner, $k$ is negative because $h=1 / 16(1+12 k)$. Hence, (26) is negative which geometrically means the approximation arc lies inside the circle. Fig. 3 shows the alternative function $\psi(t)$ of arc $Z$ for varies values of $\Phi$. It clears that the error is increasing as $\Phi$ increase.

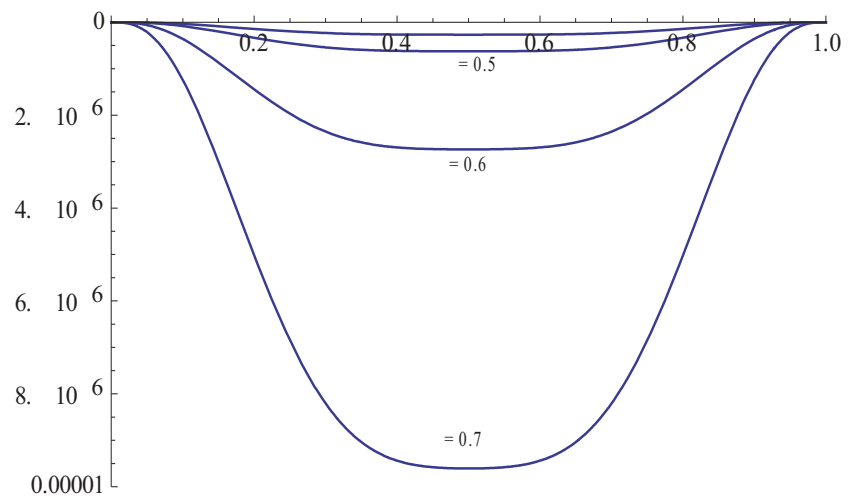

Fig. $3 \psi(t)$ for $\Phi=0.45,0.5,0.6,0.7$.
Now, if we let the uniform norm of $\psi(t)$ on $[0,1]$ which is denoted by $\|\psi(t)\|_{\infty}=\max _{t \in[0,1]}|\psi(t)|$ then at $t=1 / 2$ we have

$$
\|\psi(t)\|_{\infty}=-\frac{B k}{64}
$$

Finally, the Hausdroff distance $d_{H}(b, Z)$ can be simplified as

$$
\begin{aligned}
& d_{H}(b, Z)=\sqrt{1-\|\psi(t)\|_{\infty}}-1 \\
& \quad=\sqrt{\frac{1}{256}\left(\tau^{2}+8 \omega \cot ^{2} \Phi\right) \sec ^{2} \Phi \tan ^{4} \Phi+1}-1
\end{aligned}
$$

Next, by using MATHEMATICA, we obtain that the approximation order of $Z$ for the circular arc $b$ from Taylor expansion at $\theta=0$ is

$$
d_{H}(b, Z)=\left(-\frac{17}{98304}+\frac{1}{4096 \sqrt{2}}\right) \theta^{8}+\mathrm{O}\left[\theta^{10}\right]
$$

Compare to previous best known methods, our proposed method also have the approximation of order eight. Fig. 4 illustrates the Hausdorff distance for $0 \leq \theta \leq \pi / 2$. At $\theta=\pi / 2$ we get $\left|d_{H}(b, Z)\right|=1.25 \times 10^{-5}$.

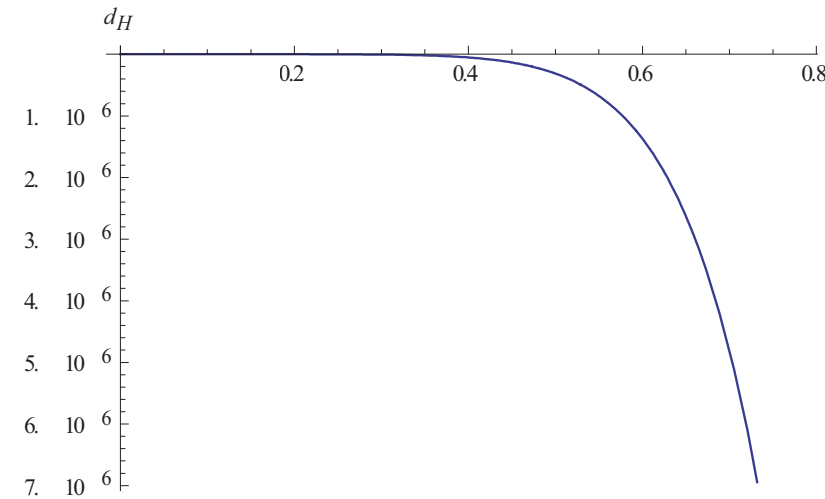

Fig. $4 d_{H}(b, Z)$ for $0 \leq \theta \leq \pi / 2$.

For a unit quarter of a circle, $\theta=\pi / 2$, other researchers such as Goldapp [5] obtained $1.96 \times 10^{-4}$ by using a cubic Bezier curve, while Ahn and Kim [1] got $3.50 \times 10^{-5}$. While using quartic approximation, Ahn and Kim [1] got $3.55 \times 10^{-6}$ and And Kim and Ahn [7] obtained $2.03 \times 10^{-6}$. Since all those results are acceptable for most practical cases, so our result can also be considered. The following is a numerical example of the approximation by the method. 


\section{NUMERICAL EXAMPLE}

Given the following data,

$$
\begin{array}{ll}
P_{0}=\left(-\frac{1}{2}, \frac{\sqrt{3}}{2}\right), & P_{4}=\left(\frac{1}{2}, \frac{\sqrt{3}}{2}\right), \\
T_{0}=\left(\frac{\sqrt{3}}{2}, \frac{1}{2}\right), & T_{1}=\left(\frac{\sqrt{3}}{2},-\frac{1}{2}\right), \quad r=1 .
\end{array}
$$

Substituting the given data into (20), (19), (15), (3) and followed by (4), we have the following results

$$
\begin{aligned}
& \alpha=0.3451, \rho=0.4585, \\
& P_{S}=(0,1.1547), P_{1}=(-0.2707,0.9984), \\
& P_{2}=(0,1.0468), P_{3}=(0.2707,0.9984)
\end{aligned}
$$

So from (1), we obtained the quartic Bezier that satisfies the given data. We illustrate this approximation curve as shown in Fig. 5, where the approximate curve is in plain arc and the standard arc circle is in dashed arc. Note that the approximation arc is coinciding with the standard arc circle.

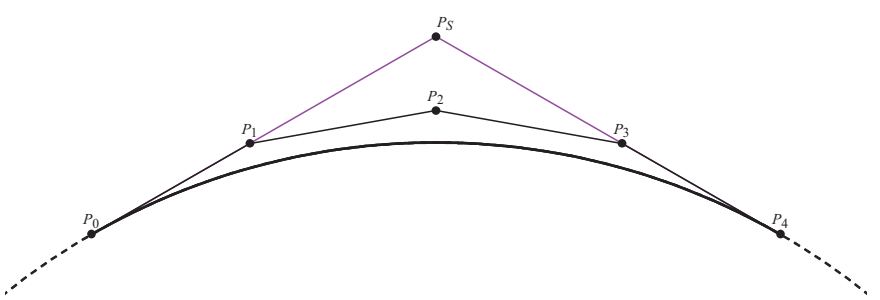

Fig. 5 An approximate curve and a standard circular arc

\section{Conclusion}

We present a method that give quick finding of an approximate curve to circular arcs by a single segment of quartic Bezier curve. An approximate curve can be straightforward blended on given two points with prescribed locations and directions on the circumference of a circle. The setting of interior control points of quartic Bezier curve is defined from a set of barycentric coordinate which geometrically understandable by users and give the curvature continuous approximation of degree two. Compared to the other previous methods, the approximation order of this proposed method is also eight. Although the order of accuracy of the proposed method is less than the best previous known results, our introduced quartic Bezier form has the advantage of easily counter the constrained $G^{2}$ Hermite interpolation problem by combination through $\mathrm{S}, \mathrm{C}$-shape of a single cubic or quartic transition curve. Also we can easily extend the result to find the spline arc, which will reduce the error of the approximation or to construct an arc of central angle $\pi \leq \theta \leq 2 \pi$.

\section{Acknowledgment}

The authors are very grateful to the anonymous referees for their valuable suggestions. This work was supported by the Ministry of Higher Education of Malaysia FRGS (2010-0066102-02).

\section{REFERENCES}

[1] Ahn Y.J. and Kim H.O. Approximation of circular ares by Bezier curves. Computer Aided Geometric Design, 81:145-163, 1997.

[2] deBoor C., Hollig K. and Sabin M. High accuracy geometric Hermite interpolation. Computer Aided Geometric Design, 4:269-278, 1987.

[3] Dokken T., Dehlen M., Lyche T and Morken K. Good approximation of circles by curvature-continuous Bezier curves. Computer Aided Geometric Design, 7:33-41, 1990.

[4] Fang L. Circular arc approximation by quintic polynomial curves. Computer Aided Geometric Design, 15:843-861, 1998.

[5] Goldapp M., Approximation of circular arcs by cubic polynomial. Computer Aided Geometric Design, 3:227238, 1991

[6] Grandine T.A and Hogan T.A. A parametric quartic spline interpolant to position, tangent and curvature. Computing (DOI), 72:65-78, 2004.

[7] Kim S.H. and Ahn Y.J. An approximation of circular arcs by quartic Bezier curves. Computer Aided Geometric Design, 30:490-493, 2007.

[8] Riskus A. Approximation of a cubic Bezier curve by circular arcs and vice versa. Information Technology and control, Vol 35, 4:371-378, 2006.

[9] Yamaguchi F. Curves and surfaces in computer aided geometric design. Springer-Verlag 1988

[10] Žagar, Emil, Jaklič, Gašper, Kozak, Jernej, Krajnc, Marjeta. Approximation of circular arcs by parametric polynomial curves. Annali Dell'Universita' di Ferrara, 53;2:271-279, 2007. 